\title{
SIMILARITY-BASED SHAPE PRIORS FOR 2D SPLINE SNAKES
}

\author{
Daniel Schmitter and Michael Unser \\ Biomedical Imaging Group, École polytechnique fédérale de Lausanne (EPFL), Switzerland
}

\begin{abstract}
We present a new formulation of a shape space containing all continuously defined 2D spline curves up to a similarity transform of a reference shape. We are able to measure a distance between an arbitrary curve and the shape space itself. Our contribution is an explicit formula for this distance measure in the continuous domain. This allows us to define efficient snake energies based on shape-dependent prior knowledge to facilitate segmentation in bioimaging. The spline-based algorithm that we propose allows us to implement continuousdomain solutions with no additional computational cost compared to the case where curves are described by a discrete set of landmarks. The proposed implementation is freely available in the public domain.
\end{abstract}

Index Terms - shape space, splines, spline snakes, active contours, similarity.

\section{INTRODUCTION}

The geometric transform called "similarity" is defined as the combination of translation, rotation, and isotropic scaling. It is particularly useful whenever one wants to segment known structures (e.g., cells, bacteria) but does not know beforehand their orientation and location in an image, a task which is often encountered in natural scenes (see Figure 1). It represents an important class of transformations if we want to characterize structures of interest [1]. In this paper, we present a formulation of the space that contains precisely all the shapes that are described by such a transformation of a reference shape. Our formulation facilitates segmentation tasks because it allows us to formulate energy terms that combine a data term with the distance between a given curve and the closest similarity transform of the reference shape that defines the shape space.

The classical approach to define shape spaces is to consider $I$ shapes that are described by an ordered set of $N$ points or landmarks in $\mathbb{R}^{2}$ [2]. Each shape is itself represented as one large vector $\mathbf{x}_{i} \in \mathbb{R}^{2 N}$, where $i \in I$. It is geometrically normalized by aligning it to a common reference in order to remove some effects of rigid transformations. Considering

This work was funded by the Swiss National Science Foundation under Grant 200020-144355.

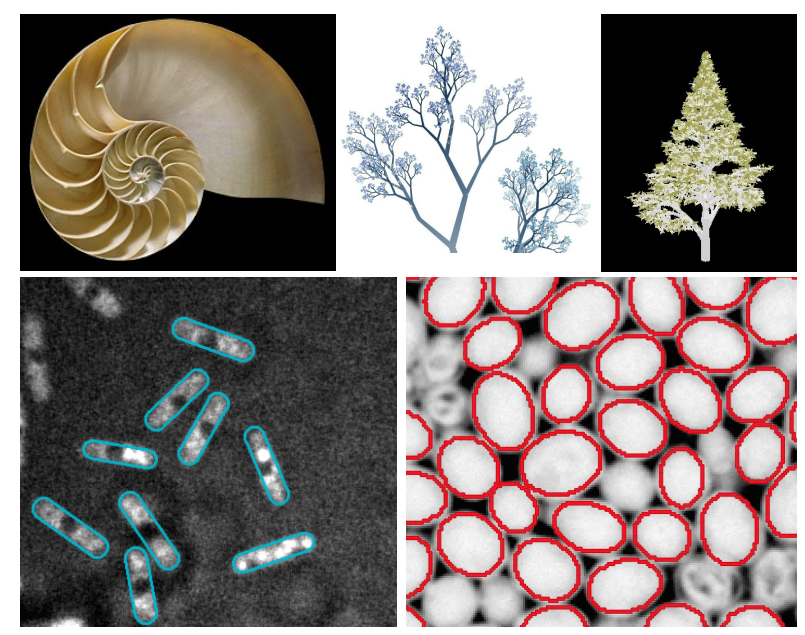

Fig. 1. Similarity occuring in nature. Top row (macroscopic world): Nautilus shell (left) and fractal-like trees (middle and right); Bottom row (microscopic world): rod-shaped (left) and oval (right) yeast cells which are all similar to each other.

a reference shape $\overline{\mathbf{x}}$ such an alignment is achieved by computing the normalized shapes as $\mathbf{y}_{i}=\min _{\mathbf{A}, \mathbf{b}}\left\|\overline{\mathbf{x}}-\mathbf{A} \mathbf{x}_{i}-\mathbf{b}\right\|^{2}$, where $\mathbf{A}$ is a transformation matrix and $\mathbf{b}$ a translation vector. Using the normalized shapes, modes of variation of the shape collection can be computed to construct a shape space.

Aside from only operating with discrete data, the classical approach has a second drawback which is the geometric normalization. By normalizing, a bias is introduced in the model because computing distances between normalized shapes usually does not yield the same result as for non-normalized shapes.

Our alternative proposal is to define a continuous-domain shape space that contains all possible similarity transforms of some reference shape. Then, searching for the minimum distance between an arbitrary shape and the shape space defined by some reference shape requires one to find the reference shape up to a similarity transform that is closest to the arbitrary shape. This idea is illustrated in Figure 2. The advantages of our method compared to the traditional approach are three-fold: 1) no normalization step is required prior to the definition of the shape space. Hence, no bias due to normal- 
ization is introduced when comparing shapes; 2) our metric takes the continuous nature of curves into account and allows us to find closed-form solutions in the continuous domain; 3 ) we propose an exact discrete spline-based implementation of the model that can be solved with no additional computational cost compared to the standard approach.

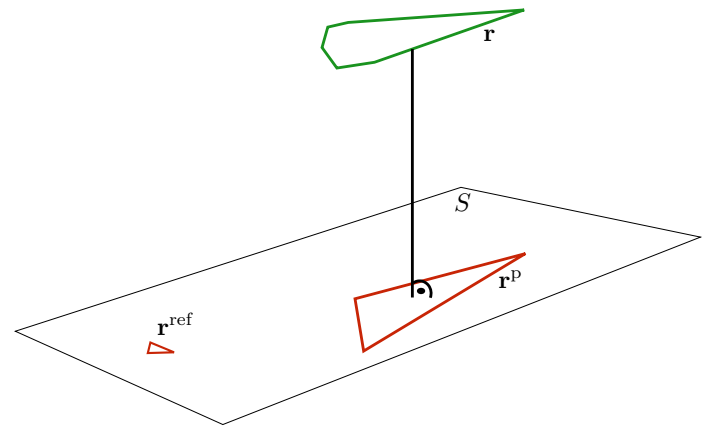

Fig. 2. Minimum distance between an arbitrary curve and a shape space. The plane denoted by $S$ represents the shape space defined by the reference shape $\mathbf{r}^{\text {ref }}$ (small red triangle). $S$ represents a shape space that contains all curves $\mathbf{r}^{\text {ref }}$ up to a similarity (i.e., rotations, scaling, and translations of $\mathbf{r}^{\text {ref }}$ ). The minimum distance between an arbitrary curve $\mathbf{r}$ (green curve) and $S$ is equivalent to the distance between the rotated, scaled, and translated triangle $\mathbf{r}^{\mathrm{ref}}$ (the large red triangle denoted as $\mathbf{r}^{\mathrm{p}}$,) that is "closest" to $\mathbf{r}$ w.r.t. a given distance measure.

\section{PARAMETRIC SPLINE CURVES}

In this section, we introduce the spline formalism that is necessary to characterize spline curves and to formulate the corresponding snake energies [3] for spline snakes [4, 5] making use of shape priors. For simplicity, we focus on 2D closed (i.e., periodic) curves because such models have a wide range of applications such as the segmentation of blob-like, elliptical, or any other kind of closed structure. The extension to open curves and higher-dimensional curves is straightforward.

\subsection{Parametric Closed Planar Curves}

Let $\mathbf{r}: D \rightarrow \mathbb{R}^{2}$ be a parametric curve such that $\mathbf{r}(t)=$ $\left(r_{x}(t), r_{y}(t)\right)$. We further assume that $r_{x}$ and $r_{y}$ are parameterized by linear combinations of suitable basis functions as

$$
\mathbf{r}(t)=\sum_{k=-\infty}^{\infty} \mathbf{c}[k] \varphi(t-k),
$$

where $\varphi$ is a compactly supported generating function based on splines and $\left\{\mathbf{c}[k]=\left(c_{x}[k], c_{y}[k]\right)\right\}_{k \in \mathbb{Z}}$ is a sequence of control points. The fact that $\mathbf{r}$ is closed can be expressed as the periodic condition $\mathbf{r}(t)=\mathbf{r}(t+1)$; hence, $D$ can be restricted to $[0,1)$. The one-dimensional functions $r_{x}$ and $r_{y}$ are also normalized to be 1-periodic. Then, we can rewrite $\mathbf{r}$ using a finite summation involving periodized basis functions as

$$
\mathbf{r}(t)=\sum_{k=0}^{M-1} \mathbf{c}[k] \varphi_{M}(M t-k),
$$

where

$$
\varphi_{M}(t)=\sum_{n=-\infty}^{\infty} \varphi(M(t-n)-k)
$$

is the $M$-periodization of the basis function $\varphi$ and the sequence of control points $\{\mathbf{c}[k]\}_{k \in[0, M-1], k \in \mathbb{Z}}$ is $M$-periodic, where $M$ coincides with the number of control points.

\subsection{Inner Product Between Curves}

By making use of the periodized basis function we derive the following inner product between the two parametric closed curves $\mathbf{r}_{1}, \mathbf{r}_{2} \in L_{2}\left([0,1], \mathbb{R}^{2}\right)$ that have the same number $M$ of control points:

$$
\begin{aligned}
\left\langle\mathbf{r}_{1}, \mathbf{r}_{2}\right\rangle & =\int_{0}^{1} \mathbf{r}_{1}(t)^{T} \mathbf{r}_{2}(t) \mathrm{d} t \\
& =\sum_{k=0}^{M} \sum_{l=0}^{M} \mathbf{c}_{1}[k]^{T} \mathbf{c}_{2}[l] \int_{0}^{1} \varphi_{M}(M t-k) \varphi_{M}(M t-l) \mathrm{d} t .
\end{aligned}
$$

By collecting the control points of each curve in the vector

$$
\tilde{\mathbf{c}}_{i}=\left[\begin{array}{c}
c_{i x}[0] \\
\vdots \\
c_{i x}\left[M_{i}-1\right] \\
c_{i y}[0] \\
\vdots \\
c_{i y}\left[M_{i}-1\right]
\end{array}\right],
$$

the inner product (1) can be expressed as

$$
\left\langle\mathbf{r}_{1}, \mathbf{r}_{2}\right\rangle=\tilde{\mathbf{c}}_{1}^{T} \mathbf{B} \tilde{\mathbf{c}}_{2}=\left\langle\tilde{\mathbf{c}}_{1}, \tilde{\mathbf{c}}_{2}\right\rangle_{\mathbf{B}}
$$

where

$$
\mathbf{B}=\left[\begin{array}{ll}
\mathbf{A} & \mathbf{0} \\
\mathbf{0} & \mathbf{A}
\end{array}\right]
$$

and $\mathbf{A}$ contains the terms related to the integrals, with $\mathbf{A}_{i, j}=$ $\int_{0}^{1} \varphi_{M}(M t-i) \varphi_{M}(M t-j) \mathrm{d} t$. Hence, the continuousdomain inner product can be efficiently computed using discrete matrix-vector operations. Therefore, $\|\mathbf{r}\|^{2}=\|\tilde{\mathbf{c}}\|_{B}$, which allows us to work with spline control points. 


\section{SPLINE-BASED SHAPE SPACE FOR SIMILARITY}

In this section we show how a shape space can be constructed that contains all shapes that are obtained by a similarity transform of an arbitrarily chosen reference spline curve. We implicitly describe the space by the continuous-domain distance between an arbitrary curve and the shape space.

\subsection{Similarity Transform}

Applying a similarity transformation (translation, rotation and scaling) to a parametric reference curve $\mathbf{r}^{\text {ref }}$ results in a new curve $\mathbf{r}^{s}=a \mathbf{R}_{\theta} \mathbf{r}^{\text {ref }}+\mathbf{b}$, where $a$ is the scaling factor, $\mathbf{R}_{\theta}$ the rotation matrix and $\mathbf{b}$ the translation vector. The next propositions state the two main results on which this paper is based. Their proof will be presented elsewhere.

Proposition 1: The curve $\mathbf{r}^{s}$ is a spline curve whose control points can be expressed as

$$
\left\{\mathbf{c}^{s}[k]=\left(\begin{array}{l}
a\left(\cos (\theta) c_{x}^{\mathrm{ref}}[k]-\sin (\theta) c_{y}^{\mathrm{ref}}[k]\right)+b_{x} \\
a\left(\sin (\theta) c_{x}^{\text {ref }}[k]+\cos (\theta) c_{y}^{\text {ref }}[k]\right)+b_{y}
\end{array}\right)\right\}_{k \in[0, M-1]}
$$

if and only if the basis function $\varphi$ satisfies the partition of unity condition $\sum_{k \in \mathbb{Z}} \varphi(t-k)=1$.

Proposition 2: The minimum $L_{2}$-distance between an arbitrary curve $\mathbf{r}$ and $\mathbf{r}^{\text {ref }}$ up to a similarity transformation and a translation is given by

$$
\begin{aligned}
& \underset{a, \theta, \mathbf{b}}{\arg \min }\left\|\tilde{\mathbf{c}}-a \mathbf{R}_{\theta} \tilde{\mathbf{c}}^{\mathrm{ref}}-\mathbf{b}\right\|_{B}^{2} \\
& =\tilde{\mathbf{c}}^{T} \mathbf{S} \tilde{\mathbf{c}}
\end{aligned}
$$

where

$$
\mathbf{S}=\mathbf{B}\left(\mathbf{I}-\mathbf{C}\left(\mathbf{C}^{T} \mathbf{B C}\right)^{-1} \mathbf{C}^{T} \mathbf{B C}\left(\mathbf{C}^{T} \mathbf{B C}\right)^{-1} \mathbf{C}^{T} \mathbf{B}\right),
$$

I is the identity matrix, $\tilde{\mathbf{c}}$ the vector of control points corresponding to $\mathbf{r}, \mathbf{B}$ is given by (2), and

$$
\mathbf{C}=\left(\begin{array}{cccc}
c_{x}^{\mathrm{ref}}[0] & -c_{y}^{\mathrm{ref}}[0] & 1 & 0 \\
\vdots & \vdots & \vdots & \vdots \\
c_{x}^{\mathrm{ref}}[M-1] & -c_{y}^{\mathrm{ref}}[M-1] & 1 & 0 \\
c_{y}^{\mathrm{ref}}[0] & c_{x}^{\mathrm{ref}}[0] & 0 & 1 \\
\vdots & \vdots & \vdots & \vdots \\
c_{y}^{\mathrm{ref}}[M-1] & c_{x}^{\mathrm{ref}}[M-1] & 0 & 1
\end{array}\right)
$$

The matrix $\mathbf{S}$ implicitly characterizes the shape space of all similarity transformations of $\mathbf{r}^{\text {ref }}$ because (3) always yields the minimum (continuous-domain) distance of an arbitrary curve $\mathbf{r}$ onto that space. Note that the matrix $\mathbf{S}$ does not depend on $\mathbf{r}$ and can be precomputed based on $\mathbf{r}^{\text {ref }}$, which allows for a fast implementation of the algorithm.

\section{SNAKE ENERGY INCLUDING SHAPE PRIORS}

In this section, we describe the main use of the proposed method. It is the construction of a new snake energy that takes into account the prior knowledge formulated by the construction of the shape space of a reference curve.

\subsection{Generic Energy Formulation}

The segmentation process is formulated as an energyminimization problem. The use of the energy term is to guide the snake towards the boundary of interest. It should ensure robustness as well as enable fast optimization. The snake energy depends on three terms: 1) the data-dependent image energy $\left.E_{\text {image }} ; 2\right)$ the internal energy $E_{\text {int }}$ which favors smoothness of the boundary and other topological aspects (e.g., prevents self-intersections); and 3) the constraint energy $E_{\mathrm{c}}$ which takes user interactions into account. The overall snake energy is given by

$$
E_{\text {snake }}(\Omega)=E_{\text {image }}(\Omega)+E_{\text {int }}(\Omega)+E_{\mathrm{c}}(\Omega),
$$

where $\Omega$ stands for the snake representation through parameters (e.g. control points). The snake optimization consists in finding

$$
\Omega^{*}=\underset{\Omega}{\arg \min } E_{\text {snake }}(\Omega) .
$$

\subsubsection{Internal Energy Considering Shape Priors}

We propose to add a shape-prior-dependent energy term to the internal energy. Such a term forces the snake to remain close to the shape space by penalizing results that differ from a similarity transform of the reference curve. Using (3), it is given by

$$
E_{\text {int,prior }}=\gamma \tilde{\mathbf{c}}^{T} \mathbf{S} \tilde{\mathbf{c}}
$$

where we control the contribution of the proposed energy term by a tuning parameter $\gamma$. The proposed energy term is specific to spline curves. It only depends on the control points of the reference curve. Hence, there is no need to explicitly match a new curve to it. The distance measure is continuous although it can be described using matrix expressions, which is due to the spline-based nature of the formulation.

\section{EXPERIMENTS}

We have simulated a culture of nineteen rod-shaped cells, as shown in Figure 3. To objectively validate the quality of the proposed energy term, the cells have different sizes, intensities, and orientation. We have also simulated cell clumps to make the segmentation more challenging as can be the case 
in real settings. The snakes have been initialized with different amounts of overlap with the cells, as well as with different sizes and orientations. They were optimized with and without the prior energy term. In an additional experiment, the segmentation was repeated on an image corrupted by additive Gaussian white noise ( stdd $=25$, SNR $=0.44 \mathrm{~dB}$ ). The results shown in Figure 3 clearly indicate the advantage of using the proposed shape prior for constructing snake energies. Most cells were correctly segmented using our approach, whereas without the prior energy term more than $70 \%$ of the segmentations need to be corrected. Testing the energy term on noisy data yielded satisfactory results too, even when the initialization only corresponded to a rough overlap with the cell target. The experiments were executed on a $3 \mathrm{GHZ}$, 32 GB RAM Mac Pro. On average, the segmentation of one cell took less than $0.2 \mathrm{~s}$.

\section{IMPLEMENTATION}

The proposed framework has been implemented as a userfriendly open-source plugin available for the bioimaging platform Icy [6]. Reference curves can be drawn as spline curves in a user-interactive way, as well as saved and reloaded at runtime. The parameter describing the contribution of the energy term can be adjusted by the user, as well as the contribution of region- and contour-based terms. In the proposed implementation the user can manually correct inaccurate results. We minimize $E_{\text {snake }}(\Omega)$ using a Powell-like linesearch method. The gradient of the energy w.r.t. the control points can be easily computed analytically, which accelerates the optimization process and improves accuracy. The plugin is freely available for Windows / Linux / Mac at http://bigwww.epfl.ch/algorithms.html.

\section{CONCLUSION}

We propose a new solution for the characterization of a shape space that contains all similarity transforms of a reference shape. We give a closed form solution in the continuous domain for the distance between an arbitrary curve and the shape space. Equations for an exact spline-based implementation of the framework are provided and we show how a new snake energy term can be constructed based on prior knowledge using the notation of shape space. Our preliminary results on synthetic images simulating realistic conditions are promising and need to be confirmed on real data. An implementation of the proposed framework is freely available as an open-source plugin for Icy.

\section{REFERENCES}

[1] S. Derrode, M.A. Charmi, and F. Ghorbel, "Fourier-based invariant shape prior for snakes," in Proc. of the IEEE Int.

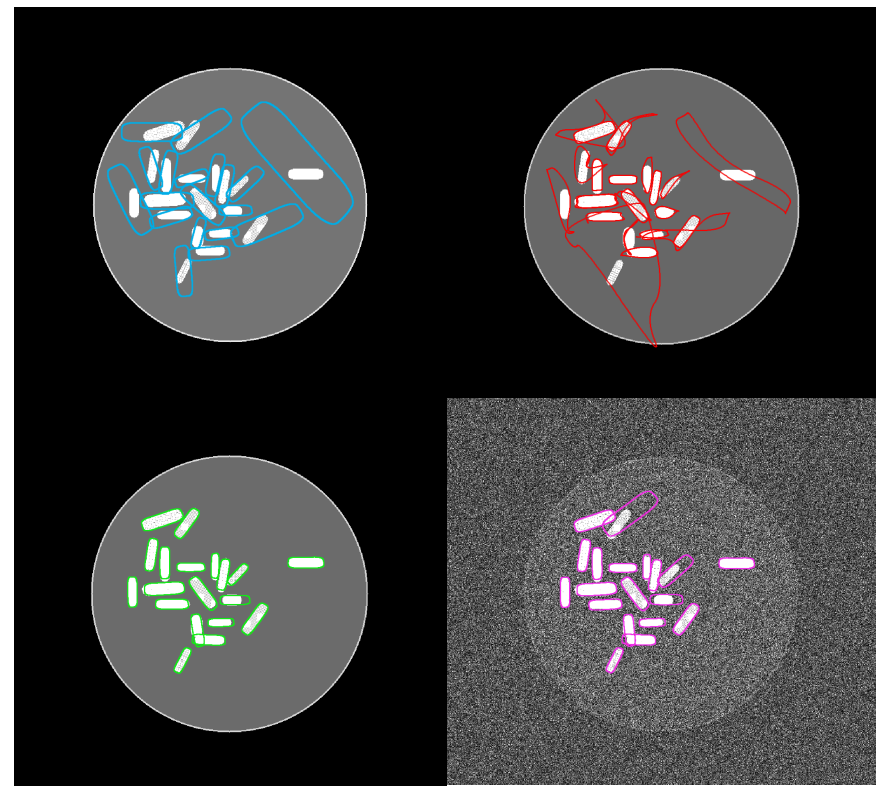

Fig. 3. Snake segmentation using shape priors. Top left: initialization of the snakes; top right: result of segmentation without shape priors; bottom left: result of segmentation with shape priors; bottom right: result of segmentation with shape priors on noisy data.

Conf. on Acoustic, Speech and Signal Processing, 14-19 May 2006, p. II.

[2] T.F. Cootes, C.J. Taylor, D.H. Cooper, and J. Graham, "Active shape models - Their training and application," Computer Vision and Image Understanding, vol. 61, no. 1, pp. 38-59, January 1995.

[3] M. Kass, A. Witkin, and D. Terzopoulos, "Snakes: Active contour models," International Journal of Computer Vision, vol. 1, no. 4, pp. 321-331, January 1987.

[4] P. Brigger, J. Hoeg, and M. Unser, "B-Spline snakes: A flexible tool for parametric contour detection," IEEE Transactions on Image Processing, vol. 9, no. 9, pp. 1484-1496, September 2000.

[5] R. Delgado-Gonzalo, P. Thévenaz, C.S. Seelamantula, and M. Unser, "Snakes with an ellipse-reproducing property," IEEE Transactions on Image Processing, vol. 21, no. 3, pp. 1258-1271, March 2012.

[6] F. de Chaumont, S. Dallongeville, N. Chenouard, N. Hervé, S. Pop, T. Provoost, V. Meas-Yedid, P. Pankajakshan, T. Lecomte, Y. Le Montagner, T. Lagache, A. Dufour, and J.-C. Olivo-Marin, "Icy: An open bioimage informatics platform for extended reproducible research," Nature Methods, vol. 9, no. 7, pp. 690-696, July 2012. 